they dealt with the significant increase in people dying from COVID-19 and this poster explores the themes that emerged by undertaking this work.

Initially a whole scale digital pivot was required as the pandemic hit: moving all existing education activity online utilising tools such as Zoom for live teaching and providing asynchronous, self-study opportunities using an online learning platform to ensure professionals could continue to be upskilled during this time. An innovative example of this was the development of a fully asynchronous product on Nurse Verification of Expected Death, a clinical need that grew exponentially during this time. The HELP team provided two bespoke webinar series, which responded to clinical needs and was developed in collaboration with a range of clinicians who were able to provide case studies and examples to secure the learning. The first webinar series in April 2020 focused on two distinct areas, supporting GPs and supporting colleagues in care homes. The team provided weekly care homes webinars and additional ECHO sessions for them. The positive feedback received resulted in further consultation to design the second wave webinar series in November which was open to all professionals in the region and included topics continuing the conversation around wellbeing along with the opportunity to contextualise dying during COVID-19 and highlighting the value of joint working during this difficult time.

This poster explores this range of activities that were undertaken during the pandemic and focuses specifically on the values that underpin the approach including the need to be responsive and flexible, collaborative, current and contextspecific in the design of these learning opportunities.

\section{P-167 EDUCATION AND TRANSCRIBING}

Katie White, Mandy Saddington. Havens Hospices, Essex, UK

\subsection{6/spcare-2021-Hospice. 183}

Background Transcribing is an important component of medicines management in the hospice, but there are differences in approaches across hospice sites. We were aware that our policies and training could benefit from development to ensure consistency of practice.

Aims

- To redesign and improve the transcribing process for transcribers within the hospice.

- To prevent and lessen medication errors.

- To re-write the transcribing policy.

- Offer education in the means of a 2.5-hour Transcribing Workshop for all transcribers, followed up with competencies to complete. Empowering the same process across the hospice settings.

- Start to measure the outcomes by auditing Medication Administration Charts (MAR).

- Offer support to transcribers.

Methods As an organisation a new policy was created on transcribing, alongside competencies. Our organisation's Practice Facilitator developed a transcribing workshop, which incorporated the competencies to be completed upon completion of the workshop. The competencies incorporated supervised practices. Following competence audits are carried out on the MAR. Medications errors are discussed at the Clinical Quality Group.
Results Transcribing workshop with a clear process has been attended by all current transcribers. Competencies are currently underway. Some transcribers required additional support. Audits of MAR are currently ongoing.

Conclusion The redesign of transcribing education has been received by all existing transcribers and the feedback is that confidence has increased, and the standard of transcribing has significantly improved. Due to the current Coronavirus pandemic, we have had some challenging times with a decreased amount of patient flow within our paediatric settings, which has limited the amount of transcribing required. Taking this into account and that the educational change is rather new, we need to wait to see if the changes we have made have made a difference to the transcribing medication errors. MAR audits will continue to remain for the foreseeable future.

\section{P-168 DESIGNING A VIRTUAL TEACHING PROGRAMME FOR HOSPICE STAFF - PHASE ONE}

Bethan Spurrier, Hazel Pearse. The Prince of Wales Hospice, Pontefract, UK

\subsection{6/spcare-2021-Hospice.184}

Background Remaining up-to-date is essential for those working in clinical settings (General Medical Council. Good medical practice. 2021; Nursing \& Midwifery Council. Standards for competence for registered nurses. 2010). However, accessing formal teaching can be challenging in the post-graduate setting, alongside delivering patient care. There is limited literature covering postgraduate education in palliative care settings in the UK.

Aim(s) We aimed to develop a teaching programme for hospice staff that could be delivered virtually, to engage part-time workers. This initial phase of the project included medical staff only, with a view to expanding once the programme was formed and well established.

Methods This pilot programme comprised three elements. Firstly, a series of 20-30 minute Zoom sessions. The sessions provided summaries of key, evidence-based information on palliative care-related topics. In addition, an online platform, called a 'Padlet'(https://padlet.com/), was used to create an online learning environment. The 'Padlet' included single-page summaries of teaching sessions, links to further learning opportunities, podcasts and conferences. Finally, the summaries were circulated via email. Sessions took place on alternate Mondays and Thursdays, to maximise attendance by parttime staff.

Results The sessions were positively received. Due to their brevity, the sessions could be incorporated into the working day easily. Zoom and 'Padlet' were both free platforms, which ensured that the programme was cost-neutral. Participants found the 'Padlet' useful and liked the single-page summaries. Challenges for the programme included: Monday being a particularly busy day clinically, and initial technical difficulties with Zoom.

Conclusions Overall this project demonstrates how teaching can be delivered in short episodes via a variety of online platforms, to engage both full-time and part-time staff. This has worked well and we are currently formally reviewing the programme to shape the next iteration of the programme. This will include inviting other members of the multidisciplinary and expanding the learning media used. 